\title{
Gait changes in a line of mice artificially selected for longer limbs
}

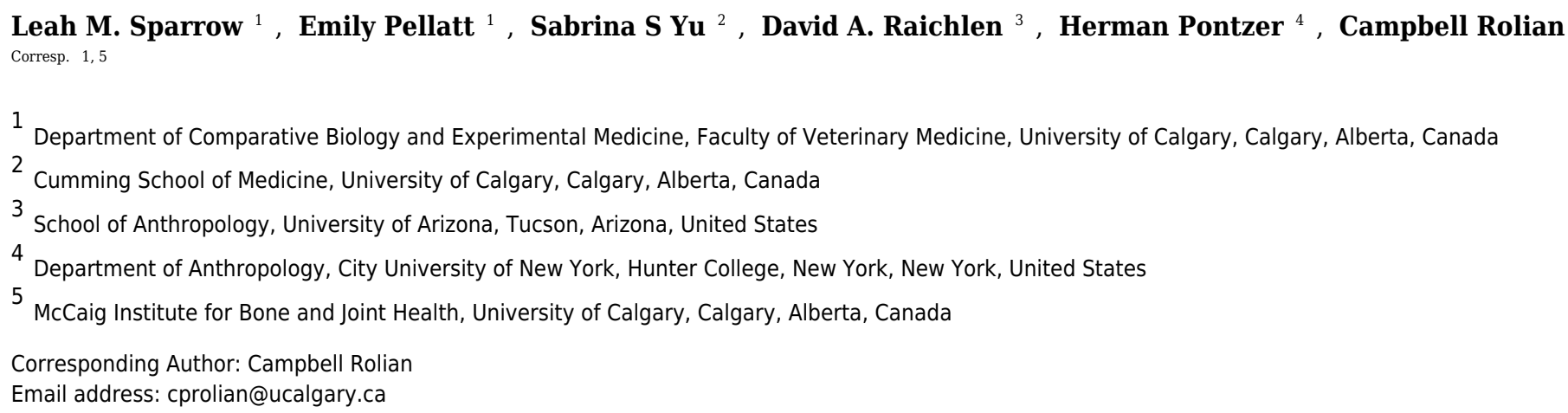

In legged terrestrial locomotion, the duration of stance phase, i.e., when limbs are in contact with the substrate, is positively correlated with limb length, and negatively correlated with the metabolic cost of transport. These relationships are well documented at the interspecific level, across a broad range of body sizes and travel speeds. However, such relationships are harder to evaluate within species (i.e., where natural selection operates), largely for practical reasons, including low population variance in limb length, and the presence of confounding factors such as body mass, or training. Here, we compared spatiotemporal kinematics of gait in Longshanks, a long-legged mouse line created through artificial selection, and in random-bred, mass-matched Control mice raised under identical conditions. We used a gait treadmill to test the hypothesis that Longshanks have longer stance phases and stride lengths, and decreased stride frequencies in both fore- and hind limbs, compared with Controls. Our results indicate that gait differs significantly between the two groups. Specifically, and as hypothesized, stance duration and stride length are $8-10 \%$ greater in Longshanks, while stride frequency is $8 \%$ lower than in Controls. However, there was no difference in the touch-down timing and sequence of the paws between the two lines. Taken together, these data suggest that, for a given speed, Longshanks mice take significantly fewer, longer steps to cover the same distance or running time compared to Controls, with important implications for other measures of individual variation in whole-organism performance, such as the metabolic cost of transport. 
1

\section{Author Affiliations:}

7

${ }^{1}$ Department of Comparative Biology and Experimental Medicine, Faculty of Veterinary Medicine,

8 University of Calgary, Calgary, Alberta, Canada

$9 \quad{ }^{2}$ Cumming School of Medicine, University of Calgary, Calgary, Alberta, Canada

$10{ }^{3}$ School of Anthropology, University of Arizona, Tucson, Arizona, United States

$11{ }^{4}$ Department of Anthropology, City University of New York, Hunter College, New York, New York, United

12 States

$13{ }^{5}$ McCaig Institute for Bone and Joint Health, University of Calgary, Calgary, Alberta, Canada

14 Corresponding Author: Campbell Rolian

15 Email address: cprolian@ucalgary.ca 
16

17

18

19

20

21

22

23

24

25

26

27

\section{ABSTRACT}

In legged terrestrial locomotion, the duration of stance phase, i.e., when limbs are in contact with the substrate, is positively correlated with limb length, and negatively correlated with the metabolic cost of transport. These relationships are well documented at the interspecific level, across a broad range of body sizes and travel speeds. However, such relationships are harder to evaluate within species (i.e., where natural selection operates), largely for practical reasons, including low population variance in limb length, and the presence of confounding factors such as body mass, or training. Here, we compared spatiotemporal kinematics of gait in Longshanks, a long-legged mouse line created through artificial selection, and in random-bred, mass-matched Control mice raised under identical conditions. We used a gait treadmill to test the hypothesis that Longshanks have longer stance phases and stride lengths, and decreased stride frequencies in both fore- and hind limbs, compared with Controls. Our results indicate that gait differs significantly between the two groups. Specifically, and as hypothesized, stance duration and stride length are 8-10\% greater in Longshanks, while stride frequency is $8 \%$ lower than in Controls. However, there was no difference in the touch-down timing and sequence of the paws between the two lines. Taken together, these data suggest that, for a given speed, Longshanks mice take significantly fewer, longer steps to cover the same distance or running time compared to Controls, with important implications for other measures of individual variation in wholeorganism performance, such as the metabolic cost of transport. 


\section{INTRODUCTION}

In terrestrial legged locomotion, a stride is divided into two phases: stance, when the foot/paw is in contact with the ground, and swing, when the foot leaves the substrate as the limb transitions to the next stance phase. Stance duration is influenced by a number of anatomical and biomechanical factors, including limb length. Limb length is positively correlated with step length, the distance traveled while a foot is in contact with the ground. As stance duration is simply step length divided by travel speed, for a given travel speed, organisms with relatively longer limbs typically have longer stance durations [1]. Stance duration is itself an important determinant of whole-organism performance, especially in relation to the metabolic cost of moving the body. During level walking and running, muscle forces produced by the limbs, integrated over the stance phase, must equal bodyweight integrated over the whole stride. Longer stance phases reduce muscle force production rates per unit of body mass, in turn reducing the overall metabolic cost of supporting the body during locomotion [2-5]. Hence, it follows that organisms with longer limbs and stance durations also tend to have a relatively lower massspecific metabolic cost per unit of distance traveled, often called the cost of transport or COT $\left(\mathrm{J} \mathrm{kg}^{-1} \mathrm{~m}^{-1}\right)$ $[2,4]$ are well documented at the interspecific level, e.g., among terrestrial quadrupedal mammals in which limb length and COT data were sampled from the same individuals [1-4, 6-8]. In contrast, these relationships have been more equivocal at the population level, where differences among individuals in whole organism performance due to variation in these factors have the potential to lead to differential reproductive success (i.e., where natural selection operates [9]). Several studies in human populations, have shown a correlation between limb length and gait variables such as stance duration [10-12] , as well as between limb length and COT in walking and running, after controlling for other factors that 
58 influence COT, such as body mass (e.g., Refs [13-18], but see also Ref. [19]). Fewer intraspecific studies

59 have examined the relationships between hind limb length, kinematics, and COT within quadrupedal

60 species, and these are limited to comparisons among breeds of dogs or horses. Longer limbs are

61 associated with greater stance duration in dogs $[20,21]$ and horses $[22,23]$, but while hind limb length

62 can be predictive of COT in dogs [13], taller horses do not necessarily have lower COT [24].

63

64

65

66

67

68

69

70

71

72

73

74

75

76

77

78

79

One of the practical challenges in relating limb length to locomotor mechanics among

individuals within populations, is that only a limited range of limb lengths can realistically be sampled, meaning that either variation in skeletal anatomy is too small to detect subtle effects of leg length on gait (i.e., a low signal-to-noise ratio), or impractically large samples are necessary to do so.

Another important limitation of the comparative approach to studying the relationship between limb anatomy and locomotor mechanics - both within and between species - is the complexity of teasing apart the relative impact on gait of body mass, skeletal anatomy, posture and other biological factors (e.g., proportions of muscle fiber types [25]). Within species, many of these factors are genetically and phenotypically correlated, and interact with each other, and with other factors such as age, body composition (e.g., lean mass), and training, which makes it harder to quantify the relative contributions of limb length alone to gait variation among individuals. For example, among artificially bred dog and horse species, differences in size and limb length are typically correlated with other changes in body proportions and conformation that can affect whole-body kinematics and may thus influence metabolic cost $[21,22]$. Similarly, among species, fundamental differences in posture (e.g., quadrupedalism vs bipedalism, limb joint extension angles, limb design (e.g., plantigrady vs unguligrady)) and orders of magnitude of differences in body mass may also obscure the effects of limb skeletal anatomy on locomotor mechanics [26]. 

and locomotor kinematics. Longshanks mice have been selectively bred for increases in tibia length independently of body mass [27, 28]. After 14 generations, Longshanks tibiae were on average $14 \%$ longer than a random-bred wild-type cohort of the same genetic background (hereafter Controls), but average body masses were the same in both groups. By pooling Longshanks and Control mice, we have created a new population of mice in which the range of variation in limb length is artificially increased, thereby circumventing sample size and resolution issues. More importantly, in this sample the potentially confounding effects of body mass, diet, training, somatic growth, age and even genetic background, are more rigorously controlled. We used the unique Longshanks mouse to investigate the proximate relationships between limb morphology and locomotor mechanics within species. We test the hypothesis that, at a given speed, increased limb length produces predictable changes in gait parameters. Specifically, we predict an increase in stance duration and stride length, and a parallel decrease in stride frequency.

\section{METHODS}

\section{Samples}

All animal procedures were approved by the Health Sciences Animal Care Committee at the University of Calgary (protocol AC13-0077), and were conducted in accordance with best practices outlined by the Canadian Council on Animal Care. We used a sex-balanced sample of adult mice (156 \pm 17 days, mean $\pm S D$ ) selected at random from 12 Longshanks families (hereafter $L S, n=11$ females, 11 males) and 12 Control families (hereafter $\mathrm{C}, \mathrm{n}=12$ females, 11 males) lines at generation F14. Details of the selective breeding protocol are given elsewhere [27]. Mice were housed individually in a separate room but in similar conditions to the main colonies of the selective breeding experiment $\left(22-25^{\circ}, 50-\right.$ 70\% humidity, 12-hour light/dark cycle). Mice were given food and water ad libitum. 
103

104

105

106

107

108

109

110

111

112

113

114

115

116

117

118

119

120

121

122

123

124

\section{Gait data collection and analysis}

a) Gait treadmill procedure

Training phase: Beginning at 10 weeks old, each mouse completed a total of eight training trials on a closed chamber treadmill (Columbus Instruments, Columbus, $\mathrm{OH}$ ) over the course of five months. In the first two trials, the treadmill was inactive, as these trials allowed the mice to acclimate to the novel treadmill environment. For the remaining six trials, mice completed an initial 5-10-minute habituation period, followed by a running schedule as follows: four minutes at $5 \mathrm{~m} / \mathrm{min}$, three minutes at $10 \mathrm{~m} / \mathrm{min}$, three minutes at $15 \mathrm{~m} / \mathrm{min}$, three minutes at $20 \mathrm{~m} / \mathrm{min}$, and a 90 second cool-down period.

Within three days of completing the final training trial, gait data for each mouse were collected using a TreadScan gait treadmill (Cleversys, Reston, VA). Mice were weighed to the nearest $0.01 \mathrm{~g}$ prior to being placed in the treadmill. The TreadScan apparatus comprises a transparent, variable-speed treadmill belt (190 $\mathrm{mm}$ long, $38 \mathrm{~mm}$ wide) enclosed within a Plexiglas chamber. While mice run on the treadmill, their paw placements are recorded on the ventral surface through the use of a mirror placed at 45 degrees to a high-speed camera recording at 100 frames per second. To improve digital tracking of footprints, the contrast between paws and fur was enhanced by applying red food coloring to the plantar surfaces of the paws. The mice were run for approximately three minutes during which three 20second digital video recordings were taken at each of three test speeds: 10,15 , and $20 \mathrm{~m} / \mathrm{min}$. After the first video was captured, each foot was manually traced for approximately 20 frames (equivalent to a full locomotor cycle) to create a foot model for all subsequent analyses.

b) Gait variables and analysis 
subsequently generates multiple gait variables from the video footage. A Matlab script (v.2012b, Reston,

127 MA) was first used to extract gait variables from the TreadScan output spreadsheet from all individual strides recorded for each mouse at each speed. For consistency, and because lighting and visibility of kinematics were better for the animal's right side, only gait variables for the right forelimb and hind limb paws were used for analysis. The touch-down sequence was recorded for all four paws (see below).

Swing Time (ms): time during which that foot is not in contact with the treadmill, (3) Stride Length (mm): the treadmill, stride length is estimated as the product of stride time (i.e., stance time plus swing time) and treadmill belt speed (4)], and Stride Frequency (Hz): 1/mean stride duration. To determine whether the increased limb length of Longshanks resulted in a change in its gait sequence relative to Controls, we also obtained timing data for each paw during a full stride cycle. Specifically, using the initiation of stance in the right forepaw as a reference, we obtained the relative timing of touch-down for the other paws, expressed as a percentage of a full stride cycle. in each individual's right fore- and hind limb paws, based on one or more sets of at least three consecutive steps. Outliers, defined as data points that were greater than two standard deviations away from their respective means, were removed and the mean was recalculated. Individuals' gait variable means were based on a minimum of 3 steps in each limb type and speed condition (mean =17 steps, range $3-63)$. Videos of the slowest speed, $10 \mathrm{~m} / \mathrm{min}$, were excluded from our analyses due to the

147 difficulty in selecting a section of video with enough consecutive strides to obtain reliable gait patterns. 
sets of generalized linear models (GLM). In the first two sets, GLMs were used to test for mean

differences between lines (LS1 vs C) and speeds ( 20 and $25 \mathrm{~m} / \mathrm{min}$ ) in each gait variable within a limb

type. In the third set of GLMs, we compared mean fore- and hind limb gait variables between the mouse

lines at the fastest speed. Finally, we used a GLM to test for mean differences between lines and speeds in gait sequence variables. In all GLMs, we first used a full factorial model, in which line, speed or limb type were treated as categorical predictors, and body mass was included as a continuous predictor (covariate). The models included interaction terms for the respective categorical factors (i.e., Line $x$ Speed, or Line x Limb Type), as well as covariate-by-factor interaction terms (i.e., homogeneity of slopes significant, i.e., the effect of the categorical factors (e.g., speed) on the responses (gait variables) was not dependent on the magnitude of body mass. As argued by Engqvist [29], in the absence of significant covariate-by-factor interaction terms, GLM analyses should be re-run without these terms, as failing to exclude them implies that the main effects of the factors cannot be generalized over the range of the covariate. Conversely, however, a significant covariate-by-factor interaction term indicates that any significant mean differences among the factors are only true at the intercept, i.e., where the covariate body mass in this case - is equal to 0. body mass in the timing of touch-down of the left hind paw relative to the right forepaw $(F(7,82)=4.04$, $p=0.047)$. However, the inclusion of this interaction term had only a small effect on the least squares 
172 to be consistent with the remaining analyses, we excluded all covariate $\mathrm{x}$ factor interactions from this

173 GLM. In all GLM analyses, relevant post-hoc pairwise comparisons on all gait variables were made using

174 Tukey's HSD tests. All analyses were carried out in Statistica software (v.10.0, Statsoft, Tulsa, OK).

175

176

177

\section{Morphometric Data Collection and Analysis}

After completing the gait trials, mice were euthanized by $\mathrm{CO}_{2}$ inhalation, weighed and immediately frozen at -20C. As part of an ongoing digital tomography archive of the Longshanks experiment, full body micro-CT scans were made using a Skyscan $1173 \mu \mathrm{CT}$ scanner at a resolution of 45 $\mu \mathrm{m}(65-70 \mathrm{kV}, 75-105 \mu \mathrm{A})$. One of the Longshanks bodies was not recovered following euthanasia. 3D isosurfaces of the scans were produced using Amira v.5.4.2 (Visage Imaging, Berlin, Germany). Bone lengths were determined by calculating the linear distance between 3D digital landmarks placed on specific anatomical features of the limb long bones. Micro-CT measurements are superior to linear measurements from dissected limbs, as soft tissues are not visible on the $\mu \mathrm{CT}$ scans, and the placement of digital landmarks on homologous anatomical features across individuals is highly repeatable [28]. The following anatomical features were used for landmark placements: (1) humerus length from the center of the proximal articular surface to the distal-most point on the medial epicondyle, (2) ulna length - from the tip of the olecranon process to the tip of the styloid process, (3) carpometacarpus - from the proximal articular facet of the central carpal to the tip of the distal articular facet of the third metacarpal, (4) scapula length - from the caudal end of the spine of the scapula to the tip of the center of the glenoid (5) third manual proximal phalanx - from the center of the proximal to the center of the distal articular facets, (6) femur length - from the center of the medial condyle to the tip of the greater trochanter, (7) tibia length - from the anterior-most lip on the proximal epiphysis to the most distal point on the medial malleolus, (8) tarso-metatarsus - from the proximal dorsal border of the centrale tarsal bone to the dorsal border of the distal articular facet of the third metatarsal, (9) third 
195

196

197

198

199

200

201

202

203

204

205

206

207

208

209

210

211

21

213

214

215

pedal proximal phalanx - from the center of the proximal to the center of the distal articular facets. Limb bone lengths were also summed within limb to obtain forelimb and hind limb anatomical lengths. Twotailed t-tests were used to compare mean body mass and limb bone lengths between the groups.

\section{RESULTS}

Morphometric differences

Mean body mass was not significantly different between Control and Longshanks mice in either the test trials or the ex vivo CT scanning (Table 1). In the forelimb, all bones were significantly longer in Longshanks when compared to Control mice. Longshanks scapulae were on average $6.6 \%$ longer, humeri were $\sim 12.5 \%$ longer, ulnae $\sim 10.7 \%$, and the hand bones (carpo-metacarpus and third proximal phalanx) were $\sim 4 \%$ longer. When summed across the elements, the anatomical length of the forelimb in Longshanks, including the scapula [30,31] was on average $9.1 \%$, or approximately $4.2 \mathrm{~mm}$, longer than Control forelimbs. Hind limb bones were all significantly longer in Longshanks mice compared to the Control mice: Longshanks mice had a 7.4\% longer femur, 14.3\% longer tibia (Figure 1), $9.5 \%$ longer tarso-metatarsus, and 9.3\% longer pedal proximal phalanx (Table 1). When summed across elements (femur + tibia + foot elements), the Longshanks hind limb was on average $10.7 \%$, or $5.2 \mathrm{~mm}$, longer than Control hind limbs. Selection for relative tibia length in Longshanks thus caused disproportionate changes in the other limb bones, such that overall the Longshanks forelimb increased in length slightly less than the hind limb, although the mean difference in length between the limbs is not significantly different between Longshanks (mean $\pm \mathrm{SD}=2.84 \pm 2.85 \mathrm{~mm})$ and Control $(1.89 \pm 2.08 \mathrm{~mm})$ (Table 1, t-test, $t(42)=1.27, p=0.21)$

\section{Gait differences}


was not significantly correlated with any gait variable in the forelimb, but in the hind limb was significantly negatively correlated with swing duration, and positively correlated with stance duration

(Table 2). Both speed and line type had significant effects on forelimb and hind limb stance duration, stride length and stride frequency (Table 2, all standardized slopes significantly different from zero, $p<0.001)$. In the forelimb, speed had a significant effect swing duration, while in the hind limb there was no change in swing duration due to speed (Table 2). There was no significant interaction between line type and speed in either limb, indicating that running faster did not affect gait variables in Longshanks and Control mice differently $(0.19<p<0.92)$. significantly longer, by $10.7 \%$ in the forelimb and $9.1 \%$ in the hind limb in Longshanks compared to Controls (Table 3, Figure 2). At the faster speed, the difference between lines in mean forelimb stance duration (7.5\%) trended towards significance (Tukey's HSD, p=0.087), while in Longshanks, hind limb stance duration was over $12.7 \%$ longer (Tukey's HSD, $p<0.001$ ). Swing durations did not differ between the lines in either limb, however forelimb swing times were significantly shorter at the faster speed (Table 3, Figure 2). Mean forelimb and hind limb stride lengths are greater by $7-8 \%$ in Longshanks at both speeds, while mean stride frequency in both limbs at both speeds is decreased by $7-8 \%$ in Longshanks.

Line and limb effects on gait within speed: The GLM analysis with limb type and line as factors 
239 in Longshanks, stance duration as a percentage of stride duration (i.e., duty factor) is different in the

240 forelimb and hind limb, but fore- and hind-limb cycles are of equal duration.

Line and speed effects on gait sequence: The mean relative timing of paw touch-downs is shown

242 in Table 5, and gait sequences are shown in Figure 3. The GLM analysis showed no effect of line on gait

243 sequence data, but speed had a significant effect on the gait sequence of the forepaws (standardized

244 beta $\left.=-0.23, F_{4,85}=4.21, p=0.03\right)$, with contact of the contralateral forepaw occurring $4-5 \%$ earlier at the

245 faster speed in both lines (Tukey's post-hoc HSD, $p=$ n.s.). Combining the touch-down sequence and

246 stance duration data at $20 \mathrm{~m} / \mathrm{min}$, both lines have very similar gait sequence profiles, although

247 Longshanks mice have relatively longer hind limb stance phases (greater duty factors, Figure 3).

\section{DISCUSSION AND CONCLUSION}

Gait is influenced by a number of anatomical (e.g., mass, skeletal size) and biomechanical

factors (e.g., speed, bipedal vs. quadrupedal locomotion). Limb length is positively correlated with step

length, and hence with stance duration, in terrestrial species across a broad range of body sizes and limb challenge of sampling adequate variation in limb bone lengths that is not also correlated with other variables that influence gait..

We used the long-limbed Longshanks mouse line to test the hypothesis that these mice would have longer strides, longer stance durations and thus lower stride frequencies when compared to mice from a random-bred control group. Crucially, these two groups have been raised under the same conditions and were trained on the treadmill using identical protocols. In addition, they have the same average body mass, and come from the same genetic background. Hence, we were able to isolate the

261 training, speed and body mass. 

lower stride frequencies. At the faster speed, swing durations in the forelimb were reduced in both lines, while Longshanks' stance duration for the hind limb was longer by $12.7 \%$. Overall, however, the differences in stride length and duration, and stride frequency between the lines in both limbs remained $7-8 \%$ at $20 \mathrm{~m} / \mathrm{min}$. Interestingly, there was no difference between Longshanks and Control in swing times of either limb, at either speed. Equivalent swing times suggest that, despite its increase in bone lengths, the natural swing period of the limbs in Longshanks have not changed substantially relative to Controls, although this could also be due to greater muscle work to move the limb during the swing phase in Longshanks. Similar natural periods could result from a similar distribution of muscle mass in both lines, despite the increased limb length in Longshanks. Future work will more carefully assess why the mean swing phase durations do not differ between these populations. relative timing and sequence of stance initiation in the four limbs at $20 \mathrm{~m} / \mathrm{min}$ (Table 5, Figure 3). Moreover, the forelimb duty factors are very similar in both lines at this speed $(\sim 0.59$, Table 4$)$. However, the relatively longer stance duration of the Longshanks hind limbs at $20 \mathrm{~m} / \mathrm{min}$ increases its duty factor slightly relative to Controls ( 0.647 vs 0.612 , Table 4$)$. The reasons for the longer stance hind limb increased in length slightly more than the forelimb as a result of selection on tibia length 281 (Table 1). One limitation of this study is the absence of kinematic data from lateral views of the limbs in these mice, which precludes us from determining precisely if and how limb protraction, retraction, and 
285

286

287

288

289

290

291

292

294

295

296

297

298

299

300

301

302

303

304

305

306

307

bones (Table 1). As a first approximation, we used our morphometric and gait data to estimate

differences in joint angles in the hind limbs of Longshanks vs Controls, based on preliminary limb angular excursion data. Step length is the horizontal distance the hip travels while the paw is in contact with the substrate. On a treadmill, the proximal joints are effectively stationary, and step length represents instead the distance traveled by the paw while in contact with the treadmill belt. Stance on a treadmill can thus be modeled as a "support triangle", described by the hip joint (vertex) and the points of pawbelt contact at touch-down and toe-off (Figure 4). The base of the triangle represents step length, the height is the vertical distance between the hip and substrate. The triangle's sides represent the hind limb at touch-down and toe-off, and their lengths at these time points are determined by the limb's joint angles and bone lengths.

When the limb angular excursion (i.e., the vertex angle) is known, the lengths of the triangle's sides can be estimated. We obtained hind limb protraction and retraction angles from a small sample of Longshanks and Control mice from generation F22 ( $n=5$ each), running at $20 \mathrm{~m} / \mathrm{min}$ (Figure S1). The hind limb protraction, retraction, and excursion angles did not differ significantly between the lines (Table S1), and the overall mean excursion angle was $74.7^{\circ} \pm 2.8$ (mean $\left.\pm S E M\right)$. Thus, even though step length in Longshanks hind limbs was $12.7 \%$ longer, the angles in the support triangle in both lines were the same, implying that the hind limb support triangle's sides in Longshanks are all also $12.7 \%$ longer. Using the sine rule, we estimated the mean triangle side length at touch-down (i.e., protraction) to be $38.91 \mathrm{~mm}$ in Controls, and $43.85 \mathrm{~mm}$ in Longshanks, while at toe-off (retraction) mean lengths were $28.09 \mathrm{~mm}$ and $31.72 \mathrm{~mm}$, respectively (Figure $\mathrm{S} 1$ ).

Combining these support lengths with the mean lengths of the femur, tibia and tarsometatarsus in each line (Table 1), and assuming that the hind paw at touch-down is horizontal and at toe-off the tarso-metatarsus is perpendicular to the treadmill (Figure S1), we solved graphically for 
308

309

310

311

312

313

314

315

mean knee and ankle joints in Longshanks and Control (Figure 4). Mean angles in Longshanks hind limbs are all very similar to Controls. The greatest difference is in the knee at touch-down, which is $\sim 5^{\circ}$, or $4 \%$, more extended in Longshanks than in Control mice. This small difference may be due to the fact that the tibia and femur in Longshanks did not increase in length proportionately ( $+14.3 \%$ vs $+7 \%)$. Overall, the $10.7 \%$ increase in hind limb length in Longshanks likely accounts for much of the $12.7 \%$ increase in its hind limb step length, and necessitates only minor joint extensions at the knee and ankle in Longshanks (Figure 4). Limb angular changes in the forelimb are likely equally small, however given that the mean increase in forelimb step length $(+7.5 \%)$ is less than the overall increase in forelimb length $(+9 \%)$, the joint angular changes in that limb in Longshanks more likely entailed flexion rather than extension, absent any changes in forelimb excursion angles between the lines. More precise limb kinematics will clearly be required to confirm these data and determine the combination of changes adopted by Longshanks to increase step and stride lengths in both limbs.

Our findings have implications with respect to the cost of transport in Longshanks. The derived morphology of the Longshanks mouse altered its gait in a predictable fashion, resulting in increased stance durations and stride lengths relative to Controls. Both of these characteristics contribute to the cost of transport in terrestrial organisms. Increased stance time distributes the muscle forces necessary to support body weight over a longer interval, lowering the rate of muscle force production, i.e., the volume of muscle recruited per distance traveled [2-5]. Complementary to this, increasing stride length reduces the number of steps required to cover a given distance, reducing activation costs [4]. Thus, our gait data predict that, all else being equal, including limb and joint angles as estimated above, the massspecific cost of transport in Longshanks should be lower when compared with Control mice. We are currently testing this hypothesis with the use of a metabolic treadmill in Longshanks and Control. 
331 important driver of adaptive evolution [9, 32-34]. In the process of selecting for a target morphological

332 trait (longer tibiae relative to body mass), we have "forward engineered" a morphology in Longshanks

333 with a quantifiable impact on whole organism biomechanical performance. We do not yet know the

334 impact of the observed change in gait on physiological performance (e.g., metabolism, especially cost of

335 transport), and it is challenging to relate any differences in these measures of whole organism

336 performance to differences in survival and reproductive success of Longshanks and Control mice in the

337 wild, as these are laboratory-reared animals raised in highly controlled and homogeneous

338 environments. Nevertheless, this study demonstrates the relationship of selectable phenotypic variation

339 in skeletal anatomy to variation among individuals in whole organism performance, in the form of

340 locomotor mechanics. Hence, it provides an important link between population-level, microevolutionary

341 processes and the adaptive origins of macroevolutionary diversity in limb musculoskeletal anatomy

342 among terrestrial mammals. 


\section{ACKNOWLEDGEMENTS}

Thanks are due to Jason Anderson and Jessica Theodor for providing access to the SkyScan 1173

$\mu \mathrm{CT}$ scanner, and to Kevin Chapman in the Behavioral Core Facility in the Cumming School of Medicine

347 (University of Calgary) for assistance with the TreadScan system. John Bertram and Douglas Syme provided useful feedback at several stages of the study, and their help is gratefully acknowledged. We also wish to thank three reviewers and the associate editor, for providing feedback and suggestions on a previous version of the manuscript.

\section{FIGURE CAPTIONS}

Figure 1: $\mu \mathrm{CT}$ scans of two individuals from the study sample closest to mean raw tibia length in Control (C, $18.85 \mathrm{~mm})$ and Longshanks (LS, $21.45 \mathrm{~mm})$.

Figure 2: Comparison of gait variables in Longshanks and Control mice at 15 and $20 \mathrm{~m} / \mathrm{min}$. Boxplots of swing duration ( $A$, in milliseconds), stance duration ( $B$, in milliseconds), stride length ( $C$, in $\mathrm{mm})$, and stride frequency ( $D$, in seconds ${ }^{-1}$ ) in Longshanks (shaded box) and Control mice (open box). Horizontal lines within boxes represent medians, boxes indicate interquartile ranges, whiskers indicate non-outlier ranges, and outliers are indicated with black dots. Solid brackets below boxplots denote statistically significant mean differences between the lines within a speed, dotted brackets above boxplots indicate statistically significant differences between speeds within lines (at the $p<0.05$ level). For clarity, differences between limbs within speed are not indicated (see Table 4).

Figure 3: Mean gait sequence in Control (grey boxes) and Longshanks (thin black boxes). The solid boxes represent the stance phases of each paw, the white boxes the swing phases. Total length, from 0 to 1 , represents a full stride cycle of the right fore paw, where 0 touch-down and $1=$ touch-down of the 
366 the latter were analyzed (see methods). Dashed boxes highlight the slightly longer stance time relative

367 to stride time in the hind limbs of Longshanks. Abbreviations: $L H=$ left hind paw, LF $=$ left forepaw, RF $=$

368 right forepaw, $\mathrm{RH}$, right hind paw.

369 Figure 4: Diagram of the support triangle in Control (black) and Longshanks (red), obtained using mean

370 step length and the mean protraction, retraction and excursion angles from an unrelated sample of mice

371 from both groups ( $n=5$ each). Step length (base of the support triangle) and these angles were used to

372 obtain the lengths of sides of the support triangle in each group. Using the mean long bone lengths in

373 each group (Table 1), we then solved graphically for angles at the ankle and knee, on the assumption that

374 the tarso-metatarsus was approximately flat at touch-down, and perpendicular to the treadmill at toe-off

375 (Figure S1).

376 Figure S1: Sample video footage of a mouse running on a treadmill, showing the frame at the initiation

377 of stance for the right hind paw (A, touch-down), and the last frame before the initiation of the swing

378 phase for the same limb (B, toe-off). A stick model of the right hind limb is superimposed on each frame,

379 showing the approximate location of the hip, knee, ankle and metatarsophalangeal joints (white circles),

380 as well as the femur, tibia, tarso-metatarsus and forefoot (phalanges) (black lines). This model was used

381 to estimate the protraction angle at touch down $(P)$, and the retraction angle at toe-off (R). The sum of

382 the protraction and retraction angles is the excursion angle.

\section{TABLE CAPTIONS}

Table 1: Morphometric Data: Body masses at the gait (TreadScan) and $\mu$ CT scanning stages, and foreand hind limb bone lengths between Control and Longshanks mice, expressed as means (SEM).

387 Longshanks body was not recovered from euthanasia prior to scanning $(\mathrm{n}=21)$. 
388 Table 2: Standardized coefficients (betas, with standard errors) for each predictor variable in the linear

389 model for the gait variables in each limb. For Line, factor levels are Control = , Longshanks = 1, for

390 Speed, factor levels are $15 \mathrm{~m} / \mathrm{min}=0,20 \mathrm{~m} / \mathrm{min}=1$. Covariate-by-factor interaction terms were

391 excluded from the analyses (see Methods). The gait sequence data are shown as a fraction of the full

392 stride cycle for the right forepaw (from 0 to 1 ).

393

394

395

396

397

398

399

400

401

402

403

404

405

406

407

408

409

410

Table 3: Limb gait parameters at 15 and $20 \mathrm{~m} / \mathrm{min}$ in Longshanks ( $L S, N=22)$ vs Control (C, $N=23$ ) mice.

Data reported as least squares means \pm SEM, based on a full factorial linear model, with Line and Speed as categorical factors, and body mass as a continuous predictor (see Table 2). Significance of differences in pairwise comparisons of means were determined using post-hoc Tukey's HSD tests. Statistical significance $(p<0.05)$ of mean differences between lines within speed is indicated in bold, and between speeds within line with an asterisk. $(p<0.05)$.

Table 4: Comparison of fore- and hind limb gait parameters at $20 \mathrm{~m} / \mathrm{min}$ in Longshanks (LS, N=22) vs Control $(C, N=23)$ mice. Data reported as least squares means $\pm S E M$, based on a full factorial linear model, with Line and Limb Type as categorical factors, and body mass as a continuous predictor. Significance of differences in pairwise comparisons of means were determined using post-hoc Tukey's HSD tests. Statistical significance $(p<0.05)$ of mean differences between lines within limb type is indicated in bold, and between limb types within line with an asterisk. $(p<0.05)$.

Table 5: Comparison of gait sequences in Longshanks and Control. Data reported as means \pm SEM, based on a full factorial linear model, with Line and Speed as categorical factors, and body mass as a continuous predictor. Means represent the proportion of a full stride cycle of the right forepaw (from 0 $=$ stance initiation to $1=$ stance initiation of the next cycle) at which the other paws initiate their stance phases. No significant differences were found between lines within speed, or between speeds within line (Tukey's HSD tests). 
411 Table S1: Comparison of limb excursion angles during stance in Control and Longshanks mice. Data

412 reported as means \pm SEM (in degrees), in a sample of 10 mice unrelated to the mice used in this study.

413 The angle values for each individual were based on 4-5 steps. No significant differences were found

414 between the lines in any of the angles (two-tailed t-tests, $0.13<p<0.83$ ).

415

416

417 


\section{REFERENCES}

419 1. Hoyt, D.F., Wickler, S.J., and Cogger, E.A. (2000): "Time of contact and step length: the effect of limb 420 length, running speed, load carrying and incline". J Exp Biol 203: 221-227

421 2. Kram, R. and Taylor, C.R. (1990): "Energetics of Running - a New Perspective". Nature. 346: 265-267

422 3. Roberts, T.J., Kram, R., Weyand, P.G., and Taylor, C.R. (1998): "Energetics of bipedal running I.

423 Metabolic cost of generating force". J Exp Biol 201: 2745-2751

424 4. Pontzer, H. (2007): "Effective limb length and the scaling of locomotor cost in terrestrial animals". J Exp Biol 210: 1752-1761

5. Pontzer, H. (2016): "A unified theory for the energy cost of legged locomotion". Biol Lett. 12:

6. Taylor, C.R., Heglund, N.C., and Maloiy, G.M.O. (1982): "Energetics and Mechanics of Terrestrial Locomotion .1. Metabolic Energy-Consumption as a Function of Speed and Body Size in Birds and Mammals". J Exp Biol 97: 1-21

7. Taylor, C.R., Heglund, N.C., Mcmahon, T.A., and Looney, T.R. (1980): "Energetic Cost of Generating Muscular Force during Running - a Comparison of Large and Small Animals". J Exp Biol 86: 9-18

8. Pontzer, H. (2005): "A new model predicting locomotor cost from limb length via force production".

9. Arnold, S.J. (1983): "Morphology, Performance and Fitness". Am Zool 23: 347-361

10. Stolze, H., Kuhtz-Buschbeck, J.P., Mondwurf, C., Boczek-Funcke, A., Jöhnk, K., Deuschl, G., and Illert, M. (1997): "Gait analysis during treadmill and overground locomotion in children and adults". Electroencephalography and Clinical Neurophysiology/Electromyography and Motor Control. 105:

11. Lythgo, N., Wilson, C., and Galea, M. (2009): "Basic gait and symmetry measures for primary schoolaged children and young adults whilst walking barefoot and with shoes". Gait Posture. 30: 502-506 
441 12. Chapman, R.F., Laymon, A.S., Wilhite, D.P., Mckenzie, J.M., Tanner, D.A., and Stager, J.M. (2012):

442 "Ground Contact Time as an Indicator of Metabolic Cost in Elite Distance Runners". Med Sci Sports $443 \quad$ Exerc 44: 917-925

13. Pontzer, H. (2007): "Predicting the energy cost of terrestrial locomotion: a test of the LiMb model in humans and quadrupeds". J Exp Biol 210: 484-494

14. Cavanagh, P.R. and Kram, R. (1989): "Stride Length in Distance Running - Velocity, Body Dimensions, and Added Mass Effects". Med Sci Sports Exerc 21: 467-479

15. Steudel-Numbers, K.L. and Tilkens, M.J. (2004): "The effect of lower limb length on the energetic cost of locomotion: implications for fossil hominins". J Hum Evol 47: 95-109

16. Cavanagh, P.R. and Kram, R., (1990) "Stride length in distance running: velocity, body dimensions, and added mass effects", in Biomechanics of Distance Running, P.R. Cavanagh, Editor Human Kinetics Books: Champaign, IL. p. 35-61

17. Brisswalter, J., Legros, P., and Durand, M. (1996): "Running economy, preferred step length correlated to body dimensions in elite middle distance runners". J Sport Med Phys Fit. 36: 7-15

18. Kramer, P.A. and Sarton-Miller, I. (2008): "The energetics of human walking: Is Froude number (Fr) useful for metabolic comparisons?". Gait Posture. 27: 209-215

19. Minetti, A.E., Ardigo, L.P., Saibene, F., Ferrero, S., and Sartorio, A. (2000): "Mechanical and metabolic profile of locomotion in adults with childhood-onset GH deficiency". European Journal of Endocrinology. 142: 35-41

20. Voss, K., Galeandro, L., Wiestner, T., Haessig, M., and Montavon, P.M. (2010): "Relationships of Body Weight, Body Size, Subject Velocity, and Vertical Ground Reaction Forces in Trotting Dogs". Vet Surg 39: 863-869 
463 21. Voss, K., Wiestner, T., Galeandro, L., Hässig, M., and Montavon, P.M. (2011): "Effect of dog breed 464 and body conformation on vertical ground reaction forces, impulses, and stance times". Veterinary and Comparative Orthopaedics and Traumatology (VCOT). 24: 106-112

22. Back, W., Schamhardt, H., Van Weeren, P.R., and Barneveld, A. (1999): "A comparison between the trot of pony and horse foals to characterize equine locomotion at young age". Equine Veterinary Journal - Supplement. 30: 240-244

469

23. Galisteo, A.M., Morales, J.L., Cano, M.R., Miró, F., Agüera, E., and Vivo, J. (2001): "Inter-Breed Differences in Equine Forelimb Kinematics at the Walk". Journal of Veterinary Medicine Series A. 48: 277-285

24. Griffin, T.M., Kram, R., Wickler, S.J., and Hoyt, D.F. (2004): "Biomechanical and energetic determinants of the walk-trot transition in horses". J Exp Biol 207: 4215-4223

25. Zierath, J.R. and Hawley, J.A. (2004): "Skeletal muscle fiber type: Influence on contractile and metabolic properties". PLoS Biol. 2: 1523-1527

26. Biewener, A.A. (1983): "Allometry of Quadrupedal Locomotion - the Scaling of Duty Factor, Bone Curvature and Limb Orientation to Body Size". J Exp Biol 105: 147-171

27. Marchini, M., Sparrow, L.M., Cosman, M.N., Dowhanik, A.S., Krueger, C.B., Hallgrimsson, B., and Rolian, C. (2014): "Impacts of genetic correlation on the independent evolution of body mass and skeletal size in mammals". BMC Evol Biol. 14: 258

28. Cosman, M.N., Sparrow, L.M., and Rolian, C. (2016): "Changes in shape and cross-sectional geometry in the tibia of mice selectively bred for increases in relative bone length". J Anat 220: 940-951

29. Engqvist, L. (2005): "The mistreatment of covariate interaction terms in linear model analyses of behavioural and evolutionary ecology studies". Anim Behav 70: 967-971 
486 30. Lilje, K.E., Tardieu, C., and Fischer, M.S. (2003): "Scaling of long bones in ruminants with respect to 487 the scapula". J Zool Syst Evol Res. 41: 118-126

488 31. Schmidt, M. (2008): "Forelimb proportions and kinematics: how are small primates different from 489 other small mammals?". J Exp Bio/ 211: 3775-3789

32. Irschick, D.J. (2003): "Measuring performance in nature: Implications for studies of fitness within 491 populations". Integr Comp Biol. 43: 396-407

492

493

494

495

496

497

33. Irschick, D.J., Meyers, J.J., Husak, J.F., and Le Galliard, J.F. (2008): "How does selection operate on whole-organism functional performance capacities? A review and synthesis". Evol Ecol Res. 10: 177-196

34. Dalziel, A.C., Rogers, S.M., and Schulte, P.M. (2009): "Linking genotypes to phenotypes and fitness: how mechanistic biology can inform molecular ecology". Mol Ecol 18: 4997-5017 


\section{Figure 1}

Figure 1 - $\mu \mathrm{CT}$ scans of Longshanks and Control tibiae

$\mu \mathrm{CT}$ scans of two individuals from the study sample closest to mean raw tibia length in Control (C, 18.85mm) and Longshanks (LS, 21.45mm). 


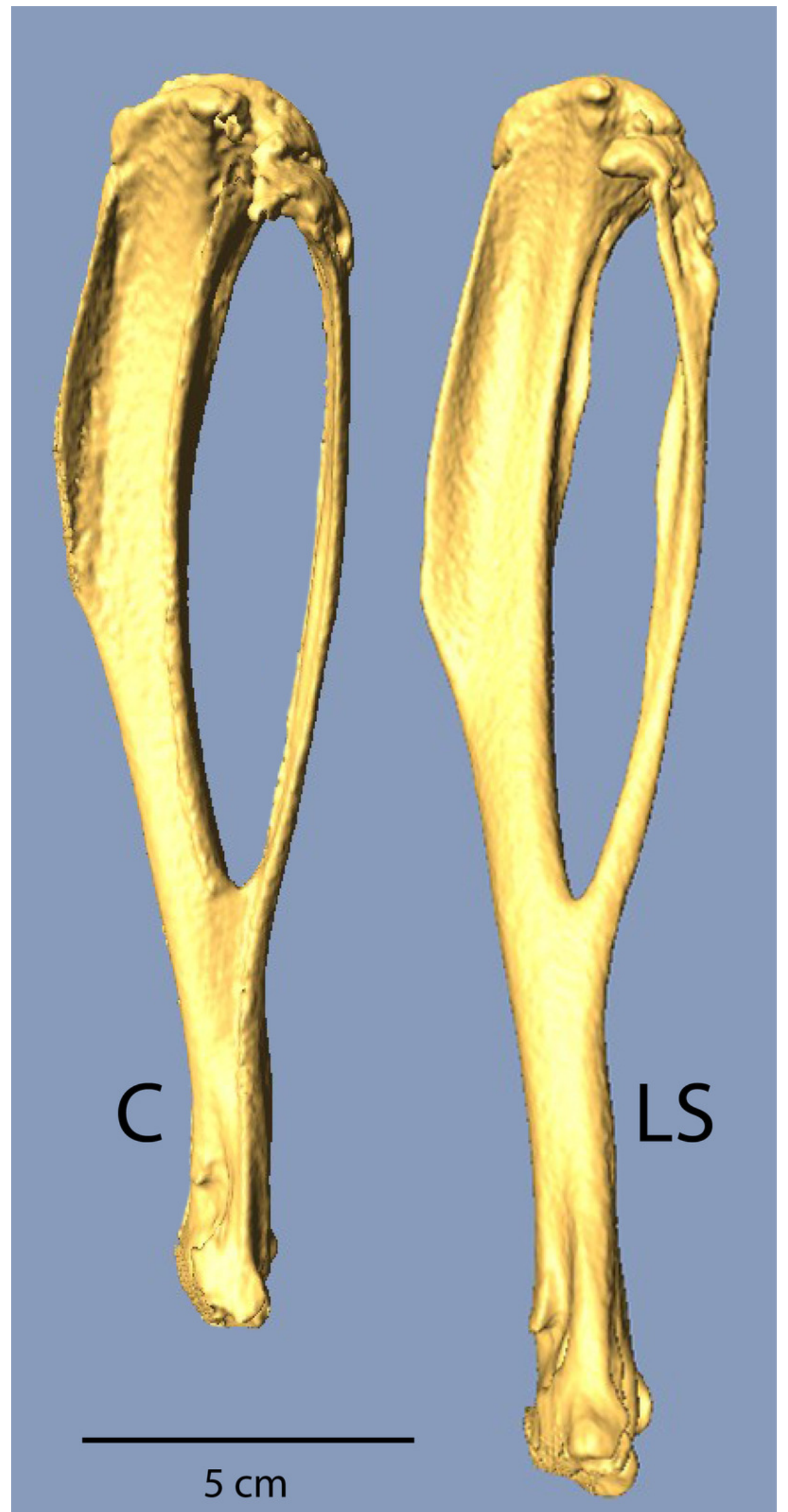




\section{Figure 2}

Figure 2 - Boxplots

Comparison of gait variables in Longshanks and Control mice at 15 and $20 \mathrm{~m} / \mathrm{min}$. Boxplots of swing duration ( $A$, in milliseconds), stance duration ( $B$, in milliseconds), stride length $(C$, in $\mathrm{mm}$ ), and stride frequency ( $D$, in seconds ${ }^{-1}$ ) in Longshanks (shaded box) and Control mice (open box). Horizontal lines within boxes represent medians, boxes indicate interquartile ranges, whiskers indicate non-outlier ranges, and outliers are indicated with black dots. Solid brackets below boxplots denote statistically significant mean differences between the lines within a speed, dotted brackets above boxplots indicate statistically significant differences between speeds within lines (at the $p<0.05$ level). For clarity, differences between limbs within speed are not indicated (see Table 4). 

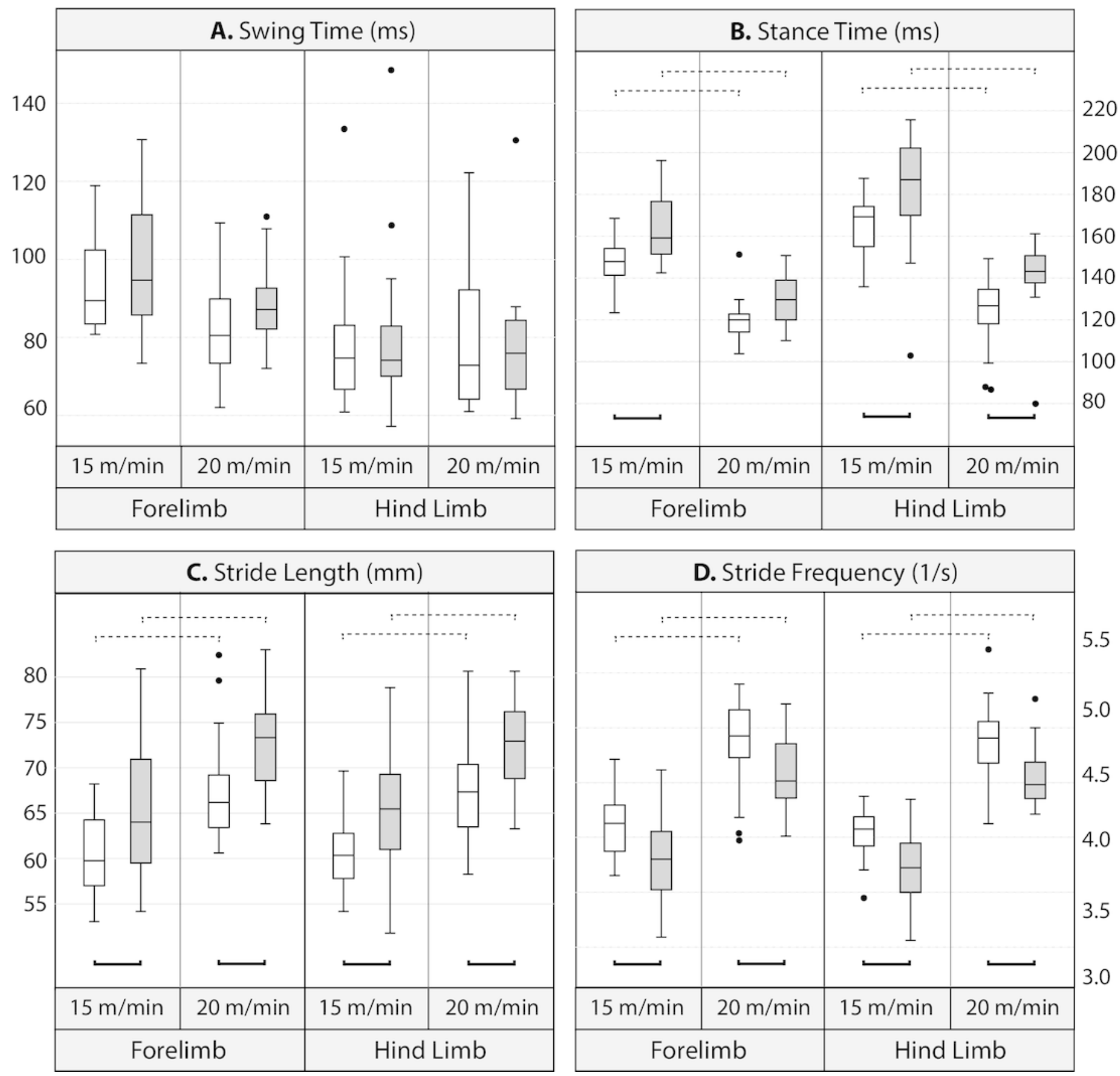


\section{Figure 3}

Figure 3 - Gait Sequence

Mean gait sequence in Control (grey boxes) and Longshanks (thin black boxes). The solid boxes represent the stance phases of each paw, the white boxes the swing phases. Total length, from 0 to 1 , represents a full stride cycle of the right fore paw, where 0 touch-down and 1 = touch-down of the following cycle. The stance phase durations of the left paws are duplicated from the right side, as only the latter were analyzed (see methods). Dashed boxes highlight the slightly longer stance time relative to stride time in the hind limbs of Longshanks. Abbreviations: $\mathrm{LH}=$ left hind paw, $\mathrm{LF}=$ left forepaw, $\mathrm{RF}=$ right forepaw, $\mathrm{RH}$, right hind paw

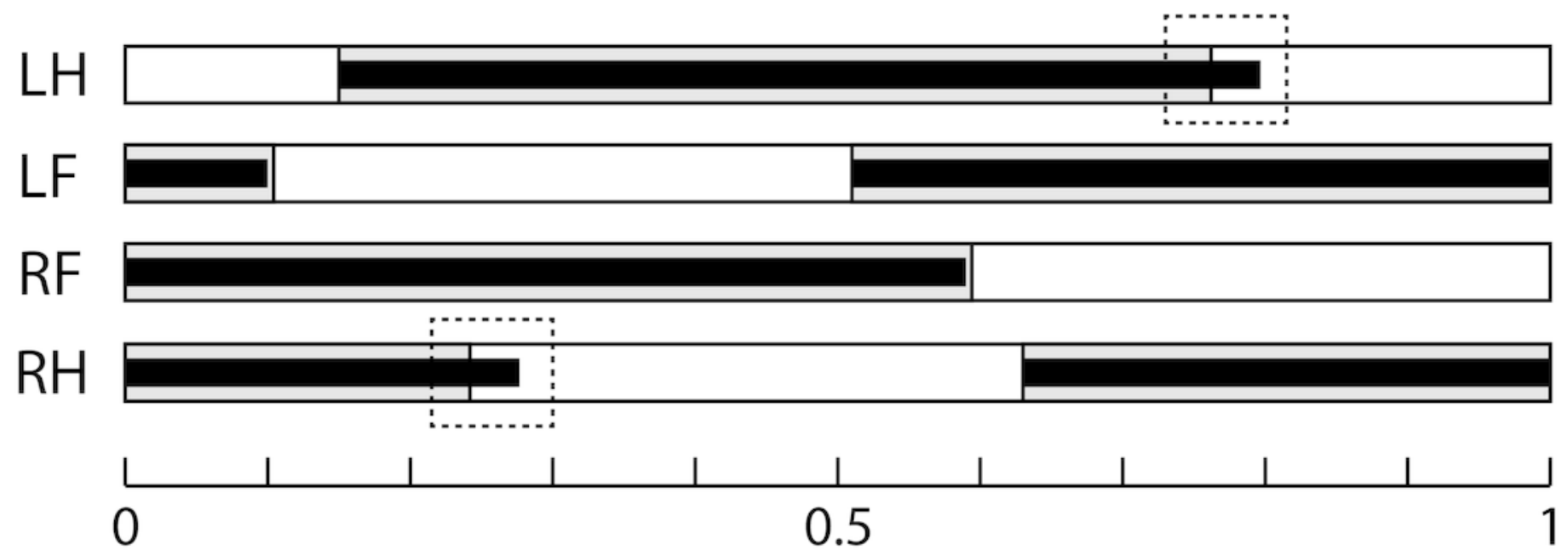




\section{Figure 4}

Figure 4 - Support Triangle

Diagram of the support triangle in Control (black) and Longshanks (red), obtained using mean step length and the mean protraction, retraction and excursion angles from an unrelated sample of mice from both groups ( $n=5$ each). Step length (base of the support triangle) and these angles were used to obtain the lengths of sides of the support triangle in each group. Using the mean long bone lengths in each group (Table 1), we then solved graphically for angles at the ankle and knee, on the assumption that the tarso-metatarsus was approximately flat at touch-down, and perpendicular to the treadmill at toe-off (Figure S1).

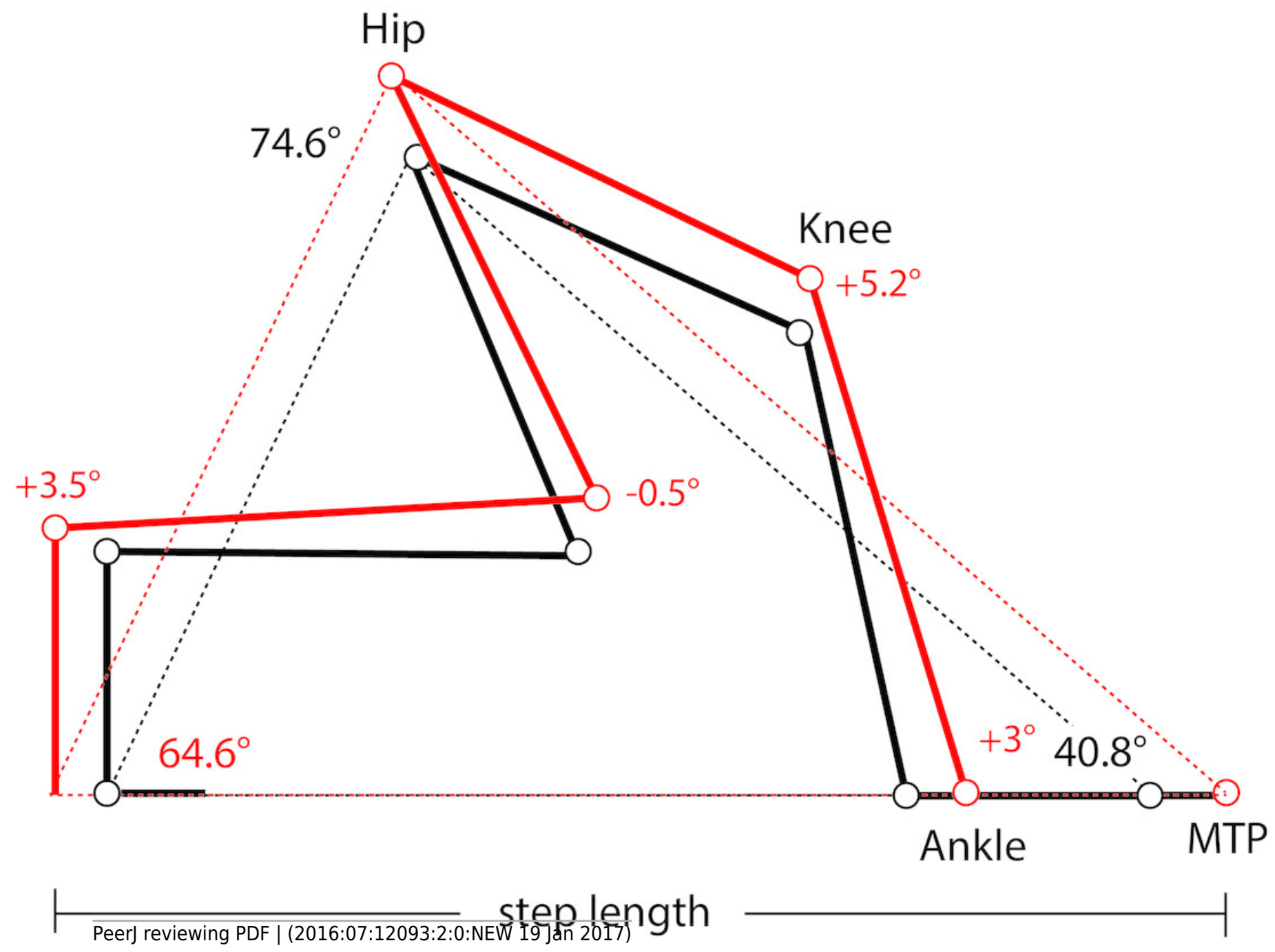




\section{Table $\mathbf{1}$ (on next page)}

Table 1

Morphometric Data: Body masses at the gait (TreadScan) and $\mu \mathrm{CT}$ scanning stages, and foreand hind limb bone lengths between Control and Longshanks mice, expressed as means (SEM). Significance of the difference in means for all variables was determined using twotailed t-tests. One Longshanks body was not recovered from euthanasia prior to scanning $(n=21)$. 
1 Table 1: Morphometric Data: Body masses at the gait (Treadscan) and $\mu \mathrm{CT}$ scanning stages, and fore-

2 and hind limb bone lengths between Control and Longshanks mice, expressed as means (SEM).

3 Significance of the difference in means for all variables was determined using two-tailed t-tests.. One

4 Longshanks body was not recovered from euthanasia prior to scanning $(n=21)$.

\begin{tabular}{|l|l|l|l|}
\hline & Longshanks $(\mathrm{n}=\mathbf{2 1})$ & Control $(\mathrm{n}=\mathbf{2 3})$ & Statistic \\
\hline Body mass (gait trials) (g) & $43.73(1.31)(\mathrm{n}=22)$ & $42.18(1.27)$ & $\mathrm{t}=0.84, \mathrm{df}=43, \mathrm{p}=0.40$ \\
\hline Body mass ( $\mathrm{CCT}$ scan) (g) & $45.81(1.43)$ & $44.55(1.60)$ & $\mathrm{t}=0.58, \mathrm{df}=42, \mathrm{p}=0.56$ \\
\hline Scapula & $13.83(0.15)$ & $12.97(0.08)$ & $\mathrm{t}=5.07, \mathrm{df}=42, \mathrm{p}<0.001$ \\
\hline Humerus & $13.65(0.11)$ & $12.17(0.07)$ & $\mathrm{t}=12.02, \mathrm{df}=42, \mathrm{p}<0.001$ \\
\hline Ulna & $17.02(0.13)$ & $15.36(0.08)$ & $\mathrm{t}=11.34, \mathrm{df}=42, \mathrm{p}<0.001$ \\
\hline Carpo-metacarpus & $4.43(0.04)$ & $4.26(0.03)$ & $\mathrm{t}=3.69, \mathrm{df}=42, \mathrm{p}<0.001$ \\
\hline Manual proximal phalanx 3 & $2.14(0.02)$ & $2.06(0.02)$ & $\mathrm{t}=2.89, \mathrm{df}=42, \mathrm{p}<0.01$ \\
\hline Femur & $18.5(0.25)$ & $17.2(0.17)$ & $\mathrm{t}=4.39, \mathrm{df}=42, \mathrm{p}<0.001$ \\
\hline Tibia & $21.44(0.33)$ & $18.75(0.18)$ & $\mathrm{t}=7.37, \mathrm{df}=42, \mathrm{p}<0.001$ \\
\hline Tarso-metatarsus & $10.62(0.17)$ & $9.7(0.10)$ & $\mathrm{t}=4.79, \mathrm{df}=42, \mathrm{p}<0.001$ \\
\hline Pedal proximal phalanx 3 & $3.35(0.06)$ & $3.06(0.03)$ & $\mathrm{t}=4.47, \mathrm{df}=42, \mathrm{p}<0.001$ \\
\hline Forelimb & $51.07(0.38)$ & $46.82(0.20)$ & $\mathrm{t}=10.11, \mathrm{df}=42, \mathrm{p}<0.001$ \\
\hline Hind limb & $53.91(0.76)$ & $48.71(0.43)$ & $\mathrm{t}=6.11, \mathrm{df}=42, \mathrm{p}<0.001$ \\
\hline
\end{tabular}

5 


\section{Table 2 (on next page)}

Table 2

Standardized coefficients (betas, with standard errors) for each predictor variable in the linear model for the gait variables in each limb. For Line, factor levels are Control $=0$, Longshanks $=1$, for Speed, factor levels are $15 \mathrm{~m} / \mathrm{min}=0,20 \mathrm{~m} / \mathrm{min}=1$. Covariate-byfactor interaction terms were excluded from the analyses (see Methods). The gait sequence data are shown as a fraction of the full stride cycle for the right forepaw (from 0 to 1 ). 
1 Table 2: Standardized coefficients (betas, with standard errors) for each predictor variable in the linear

2 model for each gait variable in each limb. For Line, factor levels are Control = , Longshanks $=1$, for

3 Speed, factor levels are $15 \mathrm{~m} / \mathrm{min}=0,20 \mathrm{~m} / \mathrm{min}=1$. Covariate-by-factor interaction terms were

4 excluded from the analyses (see methods). The gait sequence data are shown as a fraction of the full

5 stride cycle for the right forepaw.

\begin{tabular}{|c|c|c|c|c|c|}
\hline & Effect & Swing (ms) & Stance (ms) & $\begin{array}{l}\text { Stride Length } \\
(\mathrm{mm})\end{array}$ & Stride Freq. (1/s) \\
\hline \multirow{4}{*}{ 邑 } & Mass & $0.102(0.098)$ & $0.006(0.064)$ & $0.068(0.085)$ & $-0.057(0.068)$ \\
\hline & Line & $0.183(0.098)$ & $0.296(0.064)^{* *}$ & $0.362(0.085)^{* *}$ & $-0.293(0.068)^{* *}$ \\
\hline & Speed & $-0.373(0.098)^{* *}$ & $-0.752(0.063)^{* *}$ & $0.503(0.084)^{* *}$ & $0.720(0.067)^{* *}$ \\
\hline & Line*Speed & 0.051 (0.098) & $-0.082(0.063)$ & $0.010(0.084)$ & $-0.034(0.067)$ \\
\hline \multirow{4}{*}{$\begin{array}{l}\sum_{\Xi}^{\infty} \\
\underline{\underline{a}} \\
\underline{\underline{I}}\end{array}$} & Mass & $-0.308(0.104)^{*}$ & $0.2(0.065)^{*}$ & $0.023(0.084)$ & $-0.030(0.064)$ \\
\hline & Line & $0.033(0.104)$ & $0.27(0.065)^{* *}$ & $0.358(0.084)^{* *}$ & $-0.312(0.064) * *$ \\
\hline & Speed & $-0.021(0.103)$ & $-0.72(0.065)^{* *}$ & $0.522(0.083)^{* *}$ & $0.745(0.064)^{* *}$ \\
\hline & Line*Speed & $-0.06(0.103)$ & $0.006(0.065)$ & $0.015(0.083)$ & $-0.002(0.064)$ \\
\hline
\end{tabular}

6

$* p<0.05, * * p<0.001$ 


\section{Table 3(on next page)}

Table 3

Limb gait parameters at 15 and $20 \mathrm{~m} / \mathrm{min}$ in Longshanks ( $L S, N=22$ ) vs Control ( $\mathrm{C}, \mathrm{N}=23$ ) mice. Data reported as least squares means $\pm \mathrm{SEM}$, based on a full factorial linear model, with Line and Speed as categorical factors, and body mass as a continuous predictor (see Table 2). Significance of differences in pairwise comparisons of means were determined using post-hoc Tukey's HSD tests. Statistical significance $(p<0.05)$ of mean differences between lines within speed is indicated in bold, and between speeds within line with an asterisk. $(p<0.05)$. 
1 Table 3: Limb gait parameters at 15 and $20 \mathrm{~m} / \mathrm{min}$ in Longshanks (LS, $N=22)$ vs Control $(C, N=23)$ mice.

2 Data reported as least squares means \pm SEM, based on a full factorial linear model, with Line and Speed

3 as categorical factors, and body mass as a continuous predictor (see Table 2). Significant differences in

4 pairwise comparisons of means were determined using post-hoc Tukey's HSD tests. Statistical

5 significance $(p<0.05)$ of mean differences between lines within speed are indicated in bold, and between

6 speeds within line with an asterisk. $(p<0.05)$.

\begin{tabular}{|c|c|c|c|c|c|c|}
\hline & Speed & Line & Swing (ms) & Stance (ms) & $\begin{array}{l}\text { Stride Length } \\
(\mathrm{mm})\end{array}$ & Stride Freq. (1/s) \\
\hline \multirow{4}{*}{ 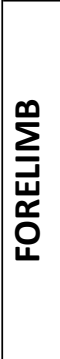 } & \multirow{2}{*}{ 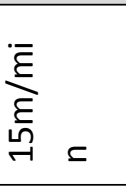 } & $\mathrm{C}$ & $94.07(2.66)^{*}$ & $147.91(2.64)^{*}$ & $60.50(1.15)^{*}$ & $4.15(0.07)^{*}$ \\
\hline & & LS & $97.67(2.72)^{*}$ & $163.8(2.7)^{*}$ & $65.35(1.18)^{*}$ & $3.87(0.07)^{*}$ \\
\hline & \multirow{2}{*}{ 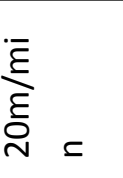 } & $\mathrm{C}$ & $82.39(2.66)^{*}$ & $119.77(2.64)^{*}$ & $67.31(1.15)^{*}$ & $4.98(0.07)^{*}$ \\
\hline & & LS & $88.83(2.72)^{*}$ & $128.75(2.7)^{*}$ & $72.45(1.18)^{*}$ & $4.61(0.07)^{*}$ \\
\hline \multirow{4}{*}{$\begin{array}{l}\sum_{\Xi}^{n} \\
\vdots \\
⿱ 亠 乂 \\
\text { I }\end{array}$} & \multirow{2}{*}{ 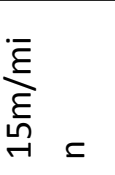 } & $\mathrm{C}$ & $77.07(3.48)$ & $166.42(3.67)^{*}$ & $60.87(1.11)^{*}$ & $4.12(0.06)^{*}$ \\
\hline & & LS & $80.25(3.56)$ & $181.57(3.76)^{*}$ & $65.44(1.13)^{*}$ & $3.78(0.07)^{*}$ \\
\hline & \multirow{2}{*}{ 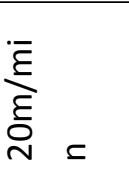 } & $\mathrm{C}$ & $78.4(3.48)$ & $124.73(3.67)^{*}$ & $67.63(1.11)^{*}$ & $4.95(0.06)^{*}$ \\
\hline & & LS & $77.5(3.56)$ & $140.55(3.76)^{*}$ & $72.61(1.13)^{*}$ & $4.60(0.07)^{*}$ \\
\hline
\end{tabular}

7 


\section{Table 4 (on next page)}

Table 4

Comparison of fore- and hind limb gait parameters at $20 \mathrm{~m} / \mathrm{min}$ in Longshanks ( $L S, \mathrm{~N}=22$ ) vs Control $(\mathrm{C}, \mathrm{N}=23)$ mice. Data reported as least squares means $\pm \mathrm{SEM}$, based on a full factorial linear model, with Line and Limb Type as categorical factors, and body mass as a continuous predictor. Significance of differences in pairwise comparisons of means were determined using post-hoc Tukey's HSD tests. Statistical significance $(p<0.05)$ of mean differences between lines within limb type is indicated in bold, and between limb types within line with an asterisk. $(p<0.05)$. 
1 Table 4: Comparison of fore- and hind limb gait parameters at $20 \mathrm{~m} / \mathrm{min}$ in Longshanks (LS, $\mathrm{N}=22$ ) vs

2 Control $(C, N=23)$ mice. Data reported as least squares means $\pm S E M$, based on a full factorial linear

3 model, with Line and Limb Type as categorical factors, and body mass as a continuous predictor.

4 Significant differences in pairwise comparisons of means were determined using post-hoc Tukey's HSD

5 tests. Statistical significance $(p<0.05)$ of mean differences between lines within limb type are indicated

6 in bold, and between limb types within line with an asterisk. $(p<0.05)$.

\begin{tabular}{|c|c|c|c|c|c|c|}
\hline $\begin{array}{l}\text { Limb } \\
\text { Type }\end{array}$ & Line & Swing (ms) & Stance (ms) & Stride (ms) & $\begin{array}{l}\text { Stride Length } \\
(\mathrm{mm})\end{array}$ & $\begin{array}{l}\text { Stride Freq. } \\
(1 / \mathrm{s})\end{array}$ \\
\hline \multirow[b]{2}{*}{$\begin{array}{l}\text { 岗 } \\
\text { ○ } \\
\text { ४ }\end{array}$} & $C$ & $82.04(3)$ & $120.12(2.76)$ & $202.16(3.32)$ & $67.31(1.11)$ & $4.97(0.07)$ \\
\hline & LS & $89.2(3.07)^{*}$ & $128.39(2.82)^{*}$ & 217.59 (3.39) & $72.45(1.13)$ & $4.61(0.07)$ \\
\hline \multirow[b]{2}{*}{ 呈 } & $C$ & $78.88(3)$ & $124.37(2.76)$ & $203.25(3.32)$ & $67.68(1.11)$ & $4.95(0.07)$ \\
\hline & LS & $77(3.07)^{*}$ & $140.93(2.82)^{*}$ & $217.92(3.39)$ & $72.56(1.13)$ & $4.61(0.07)$ \\
\hline
\end{tabular}

7 


\section{Table 5 (on next page)}

Table 5

Comparison of gait sequences in Longshanks and Control. Data reported as means \pm SEM, based on a full factorial linear model, with Line and Speed as categorical factors, and body mass as a continuous predictor. Means represent the proportion of a full stride cycle of the right forepaw (from $0=$ stance initiation to $1=$ stance initiation of the next cycle) at which the other paws initiate their stance phases. No significant differences were found between lines within speed, or between speeds within line (Tukey's HSD tests). 
1 Table 5: Comparison of gait sequences in Longshanks and Control. Data reported as means \pm SEM,

2 based on a full factorial linear model, with Line and Speed as categorical factors, and body mass as a

3 continuous predictor. Means represent the proportion of a full stride cycle of the right forepaw (from 0

4 = stance initiation to 1 = stance initiation of the next cycle) at which the other paws initiate their stance

5 phases. No significant differences were found between lines within speed, nor between speeds within

\begin{tabular}{|c|c|c|c|c|}
\hline Speed & Line & $\begin{array}{l}\text { Ipsilateral (right) hind } \\
\text { paw }\end{array}$ & $\begin{array}{l}\text { Contralateral (left) } \\
\text { forepaw }\end{array}$ & $\begin{array}{l}\text { Contralateral (right) } \\
\text { hind paw }\end{array}$ \\
\hline \multirow{2}{*}{$\frac{\stackrel{\complement}{\xi}}{\frac{\xi}{\xi}}$} & $C$ & $0.63(0.01)$ & $0.53(0.01)$ & $0.16(0.01)$ \\
\hline & LS & $0.63(0.01)$ & $0.54(0.01)$ & $0.16(0.01)$ \\
\hline \multirow[b]{2}{*}{$\frac{\stackrel{\complement}{\xi}}{\frac{\xi}{\xi}}$} & $C$ & $0.63(0.01)$ & $0.51(0.01)$ & $0.15(0.01)$ \\
\hline & LS & $0.63(0.01)$ & $0.51(0.01)$ & $0.14(0.01)$ \\
\hline
\end{tabular}

6

line (Tukey's HSD tests). 